\title{
Polarization properties of the June 21, 2001 solar corona
}

\author{
N. G. Kapanadze ${ }^{1}$, V. I. Kulijanishvili ${ }^{1}$ and Ahmed A. Hady ${ }^{2}$ \\ ${ }^{1}$ E. Kharadze Abastumani Astrophysical Observatory, Al. Kazbegi ave.2a, \\ 380060 Tbilisi, Georgia \\ email: kvazha@yahoo.com \\ ${ }^{2}$ Astronomy Dept., Faculty of Sciences Cairo University, 12613 - Giza, Egypt
}

\begin{abstract}
Five series of coronal images have been obtained by V.Kulijanishvili during the total solar eclipse of the June 21, 2001, in Zambia, Lusaka. A photographic mirror-lens coronagraphpolarimeter $(\mathrm{D}=100 \mathrm{~mm}, \mathrm{~F}=1000 \mathrm{~mm})$ was used. The absolute brightness, polarization and direction of polarization of the inner corona were measured. Standard techniques are used for the separation of the F- and K-coronas and for determination of coronal electron densities and temperatures. The background skylight polarization and intensity are calculated.
\end{abstract}

Keywords. Corona, Eclipses, Polarimeters, Hydrodynamics

We report here the results of processing the observational data obtained from the station at the Lusaka (Zambia), during the June 21, 2001 total solar eclipse by using the photographic coronagraph-polarimeter. The photographic coronagraph-polarimeter is described in detail in (Kulijanishvili et al. 1999). The corona was photographed in three positions of the polarization analyzer differing by $60^{\circ}$, and the five sets of pictures were obtained with exposure times of $3,1,1 / 4,1 / 15,1 / 30 \mathrm{~s}$. The procedure for treatment of observational material is the same as for the eclipse of the August 11, 1999 and it is described in detail in (Kulijanishvili et al. 2004, Kulijanishvili and Kapanadze, 2004).

The calibration series, consisting of ten direct photographs of the solar disk gradually decreasing in the density were obtained. The consequent calculations made to obtain the values of intensity and polarization degree of $\mathrm{K}+\mathrm{F}$ coronas are the same as that for the 11 August 1999 solar eclipse which are described in detail in (Kulijanishvili et al. 2004, Kulijanishvili and Kapanadze 2004). By using the Van de Hulst's method the separation of $\mathrm{K}+\mathrm{F}$ solar corona into $\mathrm{K}$ and $\mathrm{F}$ components was made. The obtained $I_{K}$ values of the K-corona can be checked also by van de Hulst's (1950) relation for the fraction of the $\mathrm{K}$-corona in total brightness of the maximum type corona.

For the determining the summary sky and instrumental intensity $\left(I_{A+S}\right)$ Van de Hulst's (1950) method of successive approximations was taken. The intensity value $F+I_{A+S}$ for $r=1.6 R$ was determined and according to $F+I_{A+S}$ the value of $I_{A+S}$ was estimated:

$$
I_{A+S}=4 \times 10^{-9} B \odot
$$

Van de Hulst (1950) has given a technique for deriving electron densities from measured intensities of the $\mathrm{K}$ coronal light. We have deduced electron densities from our data only in the equatorial region, where one might, with some confidence for the June 21, 2001 solar corona assume that the symmetry does exist. Figure 1a shows the resulting electron densities in the equatorial plane derived from the data on the K-corona. In Figure 1a the electron densities for the equatorial regions at maximum phase of the solar corona according to van de Hulst's (1950) model for comparison are also presented. As we can 

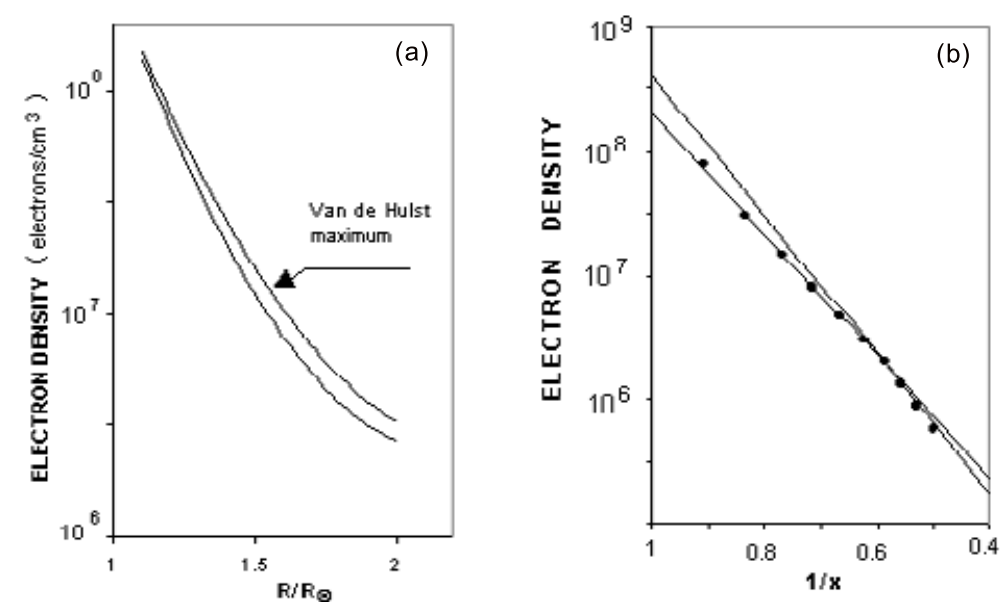

Figure 1. Panel a shows equatorial electron density and Panel b shows average equatorial electron density vs $1 / x$ in the equatorial plain of June 21, 2001 solar corona.

see from Figure 1a our values of the electron densities are in a good agreement with theoretical ones in van de Hulst's model.

Van de Hulst's model permits the existence of isothermal corona and this allowance leads to the prediction of a linear relationship between logarithm of the electron density $\ln \left(N_{e}\right)$ and $1 / x$, where $x$ is the distance from the center of the sun measured in the solar radii. Figure $1 b$ shows the plot of electron densities versus $1 / x$ in the equatorial plan of the June 21, 2001 solar corona and one can see the curvature at $r=1.5 R \odot$. It means that the model of hydrostatic distribution of the density with $T=$ const is not quite accurate and there is a temperature gradient in this area. The curvature of the relationship between $\ln \left(N_{e}\right)$ and $1 / x$ can be fitted within the accuracy of the data by assuming two linear curves, one that lies between $1.2 R$ and $1.5 R$ (curve $\mathrm{A}$ ), and the other that is between $1.5 R$ and $2.0 R$ (curve $\mathrm{B}$ ). The equations for curves $\mathrm{A}$ and $\mathrm{B}$ are:

$$
\ln \left(N_{e}\right)=9.82+\frac{9.8}{x} \quad(\text { curveA }), \quad \ln \left(N_{e}\right)=8.43+\frac{12.2}{x} \quad(\text { curveA }) .
$$

By using these equations for $r_{1}=1.3 R \odot$ and $r_{2}=1.8 R \odot$ we obtained $T_{1}=1.37 x 10^{6} \mathrm{~K}$ and $T_{2}=1.18 \times 10^{6} \mathrm{~K}$ respectively. These findings are in a good agreement with those of other researchers. In particular, it should be noted that a nonhydrostatic equilibrium behavior in the mid corona layers was as well marked in paper (Ney et al. 1961) giving the temperature gradient of $6 x 10^{5} \mathrm{~K} / R \odot$. By our estimation a proper value for the June 21, 2001 solar corona is $4 \times 105 K / R \odot$. Direction of polarization plane for the June 21 , 2001 solar corona was estimated. Obtained results show that the polarization plane is radial everywhere, except for a small deviation that does not exceed $\pm 3^{\circ}$.

\section{References}

Kulijanishvili, V. I., Ambartsumian, An. A., Borchkhadze, T. M., Goderidze, E. K., Javakhishvili, G. Sh., Japiashvili,V. P., Ozkan, M. T., Esenoglu, H. H., Kara, M., Saygac, T. 1999, in Book, Structure and Dynamics of Solar Corona, Nat. Astr. Meeting in Sol. Ph., Moscow, Troitsk, 99

Kulijanishvili, V. I., Kapanadze, N. G., Korol, A. N. 2004, Bull.of Abastumani Astrophys. Obs. 77,189

Kulijanishvili, V. I. and Kapanadze, N.G. 2005, Solar Phys. 229, 45

Hulst, H.C. van de 1950, Bull. Astron. Inst. Neth. 11, 135

Ney, E. P., Huch, W. F., Kellogg, P. J., Stein, W. and Giellet, F. 1961, ApJ 133, 616 